\title{
Confirmación histopatológica de leishmaniosis visceral en un canino de Corrientes, Argentina
}

\author{
Burna, A.N.'; Alvarez, J.D.'; Sánchez Negrette, M.; ${ }^{1}$ Maidana, H.R. ${ }^{3}$
}

Cátedras de Patología General y Sistemática ${ }^{1}$, Parasitología ${ }^{2}$ y Clínica de Pequeños Animales ${ }^{3}$, Facultad de Ciencias Veterinarias, Universidad Nacional del Nordeste, Sargento Cabral 2139, Corrientes (3400), Argentina.

Tel/fax 03783-425753. E-mail: patgral@vet.unne.edu.ar.

\begin{abstract}
Resumen
Burna, A.N.; Alvarez, J.D.; Sánchez Negrette, M.; Maidana, H.R.: Confirmación histopatológica de leishmaniosis visceral en un canino de Corrientes, Argentina. Rev. vet. 21: 2, 148-150, 2010. La leishmaniosis es una dolencia endémica registrada en más de 66 países del viejo mundo y 22 del nuevo mundo. Afecta a diferentes especies de animales domésticos y silvestres, siendo los caninos domiciliarios su principal reservorio Su agente etiológico es Leishmania chagasi, un protozoario que en los caninos produce una forma visceral con lesiones inflamatorias no supurativas en piel, hígado, intestinos, riñones, ojos y huesos. El objetivo de esta comunicación fue reportar un caso clínico con síntomas y signos característicos de la enfermedad y su diagnóstico serológico e histopatológico. A la inspección el examen clínico reveló dermatitis exfoliativa con descamación de tipo furfurácea de color plateado, hemorragias petequiales, pelo hirsuto, úlceras mucocutáneas y conjuntivitis. A la palpación se observó linfoadenopatía de los nódulos linfáticos poplíteos, preescapulares, submaxilares e inguinales. El estudio histopatológico permitió confirmar la presencia de un caso de leishmaniosis visceral canina, en un perro de la ciudad de Corrientes, proveniente de la Provincia de Formosa (Argentina). Se resalta la importancia de evitar el traslado de animales provenientes de regiones endémicas a zonas consideradas libres de leishmaniosis, como en el presente caso. El diagnóstico confirmatorio consiste, en todos los casos, en la observación de amastigotes intracelulares. Desde el punto de vista del diagnóstico de laboratorio se considera importante realizar como mínimo dos pruebas para arribar a una conclusión definitiva.
\end{abstract}

Palabras clave: perro, leishmaniosis visceral, clínica, serología, histopatología.

\begin{abstract}
Burna, A.N.; Alvarez, J.D.; Sánchez Negrette, M.; Maidana, H.R.: Histopathologic confirmation of visceral leishmaniosis in a dog from Corrientes, Argentina. Rev. vet. 21: 2, 148-150, 2010. Leishmaniosis is an endemic disease present in more than 66 countries of the old world and 22 of the new world. Many different species of wild and domestic animals may be affected. Domestic dogs are the main reservoir. The etiologic agent is the protozoan Leishmania chagasi, considered to produce the visceral form of the disease characterized by non-suppurative inflammatory lesions of the skin, liver, intestines, kidneys, eyes and bones. The aim of this work was to report a case with characteristic signs of the disease and its serological and histopathological diagnosis. The clinical examination revealed exfoliative dermatitis with silver furfuraceous scales, petequial bleedings, hirsute hair, mucocutaneous ulcers and conjunctivitis. Lymphadenopathy of popliteal, prescapular, submandibular and inguinal lymph nodes was also found. The histopathological study confirmed the presence of visceral leishmaniosis in a dog from Formosa Province in Corrientes City. It is important to avoid the transportation of animals from endemic areas to those where the disease is not present. The observation of intracellular amastigotes allows the confirmation of the disease. The need of at least two tests to perform a definitive diagnosis is emphasized.
\end{abstract}

Key words: dog, visceral leishmaniosis, clinic, serology, histopathology.

\section{INTRODUCCIÓN}

La leishmaniosis es una dolencia endémica registrada en más de 66 países del viejo mundo y en 22 paí-

Recibido: 13 agosto 2010 / Aceptado: 24 setiembre 2010 ses del nuevo mundo. Afecta a diferentes especies de animales domiciliarios y silvestres, siendo los caninos domésticos su principal reservorio. En Argentina la enfermedad es considerada importante por su impacto en la salud pública, sumado a la alta incidencia existente en países limítrofes como Brasil y Paraguay. Su 
agente etiológico es un protozoario perteneciente al orden Kinetoplastida, familia Trypanosomatidae, género Leishmania, el cual es capaz de desarrollar diferentes tipos de presentaciones clínicas: cutánea, mucocutánea y visceral ${ }^{3,7,11}$.

La etiología de la forma visceral responde al "complejo Leishmania donovani", con tres especies involucradas: Leishmania donovani y Leismania infantum en el viejo mundo y Leishmania chagasi en el nuevo mundo. Esta última es un parásito heteroxeno, que completa su ciclo de vida en dos tipos de hospedadores: vertebrados (caninos, roedores y seres humanos) e invertebrados (dípteros hematófagos de la familia Psychodidae), cuyo representante en nuestra región es Lutzomia longipalpis.

Esta parasitosis presenta un largo período de incubación, que varía de un mes a siete años. En este período los microorganismos se diseminan ampliamente, con predilección por la médula ósea, ganglios linfáticos, bazo e hígado. En los caninos se producen lesiones inflamatorias no supurativas en piel, hígado, intestinos, riñones, ojos y huesos, además del daño directo causado por la deposición de complejos inmunes en las articulaciones y en las membranas basales de los riñones, vasos sanguíneos y ojos, produciendo vasculitis, glomerulonefritis, poliartritis y uveítis ${ }^{6}$.

Los principales signos clínicos se corresponden con letargia, debilidad, pérdida de peso y lesiones características en piel. Se han observado también caninos con problemas locomotores, tales como rengueras y cojeras, debido al crecimiento exagerado de las uñas y a la poliartritis autoinmune, polimiositis y lesiones óseas ${ }^{12}$.

Los parásitos se multiplican en los macrófagos del hígado, produciendo hepatitis crónica activa y ocasionalmente, aumento palpable del hígado, vómitos, poliuria, polidipsia y anorexia. Se ha descrito colitis ulcerativa crónica con diarrea y melena ${ }^{5}$. La enteritis puede ser el resultado del daño parasitario directo (enteritis granulomatosa) o consecuencia de la insuficiencia renal. También ha descrita la pancreatitis hemorrágica aguda $^{2}$.

En los perros afectados frecuentemente se observa insuficiencia renal moderada o grave, como consecuencia de la deposición de complejos inmunes que conducen a la glomerulonefritis membranosa o extra-membranosa ${ }^{4}$. Los cuadros clínicos, aunque muy variables y poco específicos, sirven para orientar el diagnóstico, siempre y cuando se apoyen en una anamnesis exhaustiva que ofrezca datos epidemiológicos relevantes como ser hábitat, tipo de actividad, tiempo de exposición al vector, zona geográfica, procedencia y otros ${ }^{9}$. En zonas endémicas, un solo síntoma compatible debe alentar la sospecha de leishmaniosis y justificar las pruebas diagnósticas específicas. Debe considerarse sospechoso todo animal que proceda de una zona endémica $o$ que haya permanecido en la misma durante el periodo de actividad del vector ${ }^{10}$.

El diagnóstico de elección y el más fiable, es el parasitológico. La observación del parásito por visualiza- ción directa (punción de ganglios, médula ósea, biopsia cutánea), además de técnicas de biología molecular (PCR) y/o xenodiagnóstico (infección del flebótomo transmisor) son las pruebas determinantes ${ }^{1,8}$. El objetivo de la presente comunicación fue reportar un caso clínico con síntomas y signos característicos de la enfermedad y su diagnóstico serológico e histopatológico.

\section{MATERIAL Y MÉTODOS}

El caso clínico fue una perra Pit Bull de 8 meses de edad, nacida y criada en la ciudad de Clorinda, Provincia de Formosa, trasladada luego a la ciudad de Corrientes, donde vivió los dos últimos meses antes de ser sacrificada. Ante la sospecha de leishmaniosis, en el laboratorio de un consultorio veterinario privado se le extrajo sangre para realizar diagnóstico serológico, el cual resultó positivo al test $r K 39$ específico para leishmaniosis visceral canina.

Este diagnóstico fue convalidado en la Cátedra de Patología General y Sistemática de la Facultad de Ciencias Veterinarias de la UNNE, al realizarse nueva evaluación anamnésica, clínica y de laboratorio (punción de medula ósea, punción de ganglios poplíteo y preescapular, frotis sanguíneo, prueba de formogelificación y análisis bioquímico del paciente). Por solicitud del dueño se efectúa eutanasia y posterior necropsia para estudios anatomohistopatológicos. Muestras de órganos fueron fijadas en formol bufferado al 10\%, procesadas por la técnica histológica clásica, incluidas en parafina, cortadas a 5 um y coloreadas con hematoxilina y eosina.

\section{RESULTADOS}

La inspección reveló dermatitis exfoliativa con descamación de tipo furfurácea de color plateado, hemorragias petequiales, hirsutismo, úlceras mucocutáneas y conjuntivitis (Figura 1). A la palpación se constató linfoadenopatía de los nódulos linfáticos poplíteos, preescapulares, submaxilares e inguinales.

En la necropsia se observaron úlceras en el labio superior y en el paladar duro, aumento de tamaño de los nódulos linfoides submaxilares, preescapulares, poplíteos e inguinales. El examen de la cavidad abdominal reveló hepato y esplenomegalia (Figura 2), así como la presencia de una cicatriz en la cápsula esplénica. El examen de la cavidad craneana reveló aumento del líquido cefalorraquídeo.

El examen histopatológico del bazo y los nódulos linfoides evidenció infiltrado linfoplasmocitario con hiperplasia de los folículos. Los senos medulares de los nódulos linfoides presentaron abundante cantidad de plasmocitos y macrófagos, no hallándose estructuras compatibles con amastigotes de Leishmania sp. El músculo temporal presentó necrosis de fibras musculares e infiltrado inflamatorio linfocitario.

En el hígado se evidenciaron múltiples focos de infiltrado inflamatorio de tipo linfoplasmocitario, con 


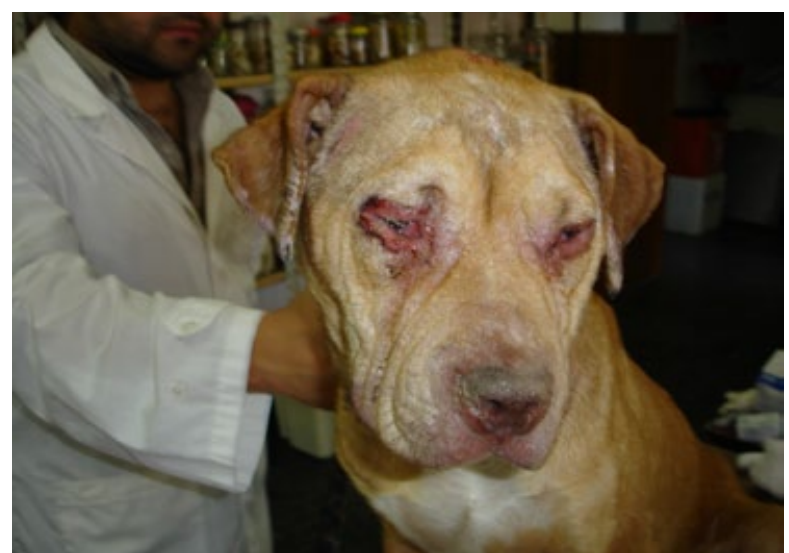

Figura 1. Severa descamación periocular y conjuntivitis.

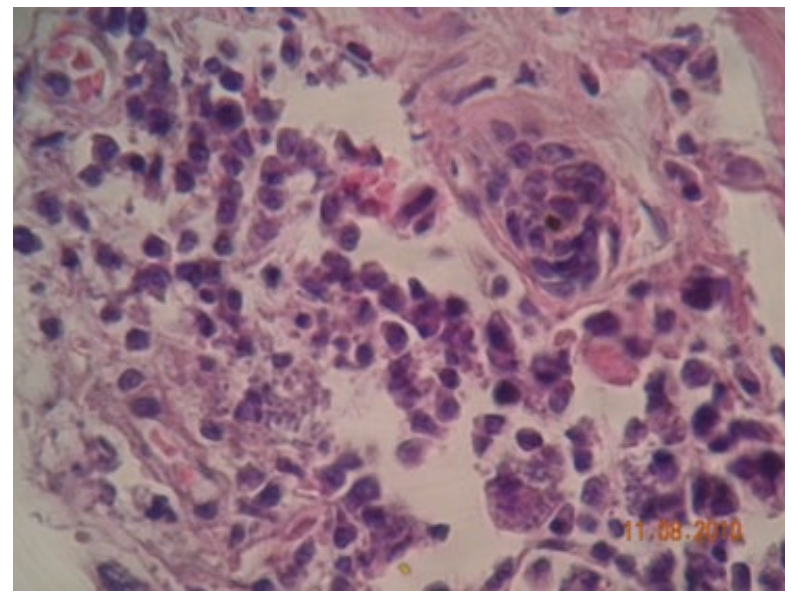

Figura 3. Piel: infiltrado inflamatorio y macrófagos con amastigotes.

degeneración hidrópica y grasa de hepatocitos y áreas de necrosis centrolobulillar. Los riñones presentaron congestión y pequeños focos de infiltrado inflamatorio linfocitario intersticial. En piel se observó abundante infiltrado inflamatorio que comprometió tanto a la dermis profunda como superficial, de tipo linfoplasmocitario, con presencia de macrófagos conteniendo escasos amastigotes de Leishmania sp. (Figura 3).

Tanto el test de formogelificación como la prueba diagnóstica con tira reactiva $r K 39$ canine kala-azar detec resultaron positivos. El examen parasitológico directo en frotis coloreados con Giemsa arrojó resultado negativo. Es importante destacar que tampoco se observaron parásitos en los extendidos de medula ósea ni nódulos linfoides. Los hallazgos de necropsia y el estudio histopatológico permitieron corroborar el diagnóstico clínico, demostrándose la presencia del protozoario y las características lesiones en varios órganos.

Se resalta la importancia de no trasladar animales de zonas endémicas a zonas consideradas libres de leishmaniosis, como en el presente caso. Además, desde el punto de vista diagnóstico se considera primordial realizar como mínimo dos pruebas para poder llegar a un diagnóstico definitivo, siendo en todos los casos la observación de amastigotes intracelulares, el diagnostico confirmatorio.

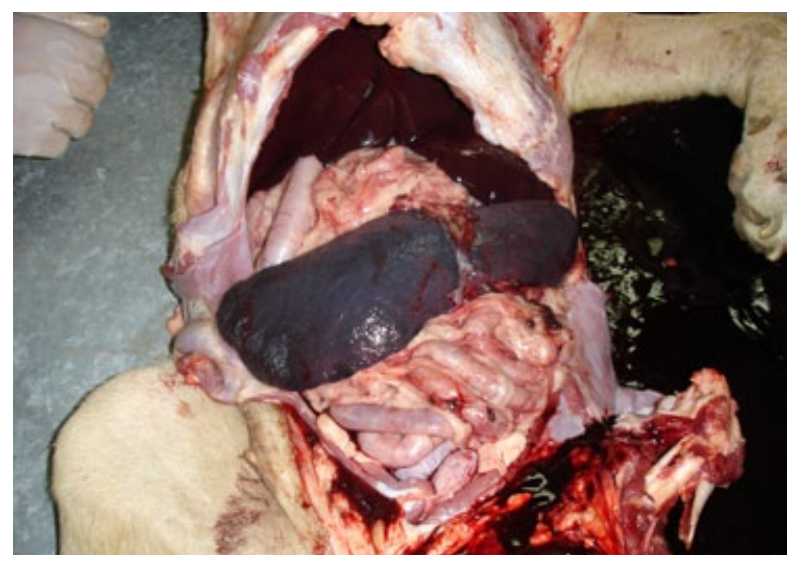

Figura 2. Hepatoesplenomegalia.

\section{REFERENCIAS}

1. Alvar J, Cañavate C, Molina R, Moreno J, Nieto J. 2002. Canine leishmaniasis. Adv Parasit 57: 1-88.

2. Carrasco L, Lara FC, Martín E. 1997. Acute haemorrhagic pancreatitis associated with canine visceral leishmaniasis. Vet Rec 141: 519-571.

3. Desjeux P. 2004. Leishmaniasis: current situation and new perspectives. Comp Immunol Microbiol Infect Dis 27: 305-318.

4. Diaz Espineira M, Slappendel R. 1997. A case of autochtthonous canine leishmaniasis in the Netherlands. Vet $Q$ 19: 69-71.

5. Ferrer L, Juanola I, Ramos A, Ramis A. 1991. Chronic cholitis due to leishmania infection in two dogs. Vet Pathol 28: $342-343$.

6. Ferrer L. 1992. Leishmaniasis. In: Current Veterinary Therapy (Kirk RW, Bonagura JD Ed), Saunders, Philadelphia, p. 266-270.

7. Gramiccia M, Gradoni L. 2005. The current status of zoonotic leishmaniasis and approaches to disease control. Int J Parasit 35: 1169-1180.

8. Killick-Kendrick R. 1999. The biology and control of phlebotomine sandflies. Clin Dermatol 17: 279-289.

9. Koutinas A, Polizopulou ZS, Saridomichelakis MN, Argyriadis D, Fytianou A, Plevaraki K. 1999. Clinical considerations on canine visceral leishmaniosis in Greece: a retrospective study of 158 cases (1989-1996). J Am Anim Hosp Assoc 35: 376-383.

10. Miró G, Fraile C. 1999. Leishmaniosis canina: revisión práctica. Rev Consulta de Difusión Veterinaria (Castellón, España) 7: 63-74.

11. Monteiro EM, Silva JC, Costa RT, Costa DC, Barata RA, Paula EV, Coelho GL, Rocha MF, Dias CL, Dias ED. 2005. Leishmaniose visceral: estudo de flebotomineos e infeccao canina em Montes Claro, Minas Gerais. Rev Soc Bras Med Trop 38: 147-152.

12. Spreng D.1993. Leishmanial polyarthritis in two dogs. $J$ Small Anim Pract 34: 559-563. 